\title{
ANTIBIOTIC CONSUMPTION IN DANISH DAIRY COWS - IS THERE A "STANDARD" TREATMENT FOR MASTITIS?
}

\author{
Bruun, J. ${ }^{1,2}$, Ersbøll, A.K. ${ }^{1,2}$, Bennedsgaard, T.W..$^{1,2}$ \\ ${ }^{1}$ The Royal Veterinary and Agricultural University, Grønnegårdsvej 8, 1870 Frederiksberg C, Denmark. \\ ${ }^{2}$ Research Centre for the Management of Animal Production and Health, Foulum, P.O. Box 50, \\ 8830 Tjele, Denmark.
}

Mastitis is believed to be the primary cause of antibiotic usage in dairy cows. Also, it has often been stated that the treatment in Denmark for mastitis occurs using primarily small spectrum penicillin. The antibiotic usage for the disease groups: mastitis, lameness, reproductive diseases, digestive diseases, and the variation in antibiotic treatment of mastitis is evaluated. Furthermore, the level of variation explained by herd, and veterinary practice is investigated.

The data was collected between January 1999 and January 2000 in 18 herds and 7 practices in a region around the Konge $\AA$ in the southern part of Jutland. Data sources included the Danish cattle database for cow information e.g. animal identification, disease treatments, and dates for disease treatment. Data on antibiotic usage was typed from accounting records, individual cow cards and milk and meat withdrawal notes. The diseases were grouped into five different groups: mastitis, lameness, reproductive diseases, digestive diseases, and other diseases. The antibiotics used were grouped into 7 different groups according to the active ingredients, only antibiotics for injection was included. For logistic regression purposes antibiotic usage for mastitis was recoded as a binary outcome of penicillin procaine usage vs. other antibiotics.

The methods of analysis used include descriptive statistics for disease groups and antibiotic consumption. The level of variation in antibiotic usage with relation to penicillin or not was investigated using multivariable logistic regression.

The preliminary results showed that $66 \%$ of the treatments with injectable antibiotics are used for treatment of mastitis. The remaining in decreasing order for treatment of lameness (12\%), reproductive diseases $(6 \%)$, suffering other and infection (6\%), and digestive disorders (5\%). For treatment of mastitis penicillin procaine was used in $47 \%$ of the cases. In order of decreasing usage were: ampicillin (13\%), oxytetracycline (10\%), streptomycin/penicillin combinations $(8 \%)$, spiramycin $(8 \%)$, trimetroprim/sulfa combinations $(7 \%)$, and amoxicillin $(3 \%)$.

In the multivariable logistic regression model the significant level $(\mathrm{p}<0.001)$ in relation to the odds for penicillin usage or not was veterinary practice, however 3 practices had to be excluded because only one herd was included within these practices. The level of herd nested to veterinary practice was non-significant.

The conclusions of the present study was that mastitis does account for much of the injectable antibiotics used, but other diseases also have to be considered when trying to decrease the amount of antibiotics used. Penicillin does seem to be the drug of choice for mastitis, but more broad spectrum antibiotics are certainly also used.

There was no standard treatment of mastitis across veterinary practices, but whether this is true once stratified for veterinary practice, lactation number, micro organisms involved, and first time mastitis vs. second or more remains to be investigated. 